\title{
SCIENTIFIC REPORTS

\section{VAR2CSA binding phenotype has ancient origin and arose before Plasmodium falciparum crossed to humans: implications in placental malaria vaccine design}

\author{
Stéphane Gangnard ${ }^{1,2,3}$, Arnaud Chêne ${ }^{1,2,3}$, Sébastien Dechavanne ${ }^{1,2,3}$, Anand Srivastava $\oplus^{1,2,3}$, \\ Marion Avril ${ }^{4}$, Joseph D. Smith ${ }^{4,5}$ \& Benoît Gamain $\mathbb{1}^{1,2,3^{*}}$
}

\begin{abstract}
VAR2CSA is a leading candidate for developing a placental malaria (PM) vaccine that would protect pregnant women living in malaria endemic areas against placental infections and improve birth outcomes. Two VAR2CSA-based PM vaccines are currently under clinical trials, but it is still unclear if the use of a single VAR2CSA variant will be sufficient to induce a broad enough humoral response in humans to cross-react with genetically diverse parasite populations. Additional immuno-focusing vaccine strategies may therefore be required to identify functionally conserved antibody epitopes in VAR2CSA. We explored the possibility that conserved epitopes could exist between VAR2CSA from the chimpanzee parasite Plasmodium reichenowi and Plasmodium falciparum sequences. Making use of VAR2CSA recombinant proteins originating from both species, we showed that VAR2CSA from $P$. reichenowi (Pr-VAR2CSA) binds to the placental receptor CSA with high specificity and affinity. Antibodies raised against Pr-VAR2CSA were able to recognize native VAR2CSA from different $P$. falciparum genotypes and to inhibit the interaction between CSA and $P$. falciparum-infected erythrocytes expressing different VAR2CSA variants. Our work revealed the existence of crossspecies inhibitory epitopes in VAR2CSA and calls for pre-clinical studies assessing the efficacy of novel VAR2CSA-based cross-species boosting regimens.
\end{abstract}

Placental malaria (PM) is a serious complication of malaria infection associated with accumulation of Plasmodium falciparum infected erythrocytes (IEs) in the placental intervillous space ${ }^{1-3}$, leading to adverse health consequences for both mother and child ${ }^{4}$. Pregnant women living in malaria endemic areas gradually acquire antibodies that limit placental infections ${ }^{5}$, thus diminishing the severe clinical outcomes associated with PM. In contrast to parasite populations sequestering in the peripheral microvasculature, which bind to endothelial cell receptors such as CD36, intercellular adhesion molecule 1 (ICAM-1) and endothelial protein C receptor (EPCR) ${ }^{6-8}$, placental IEs bind to an unusually low-sulphated form of chondroitin sulphate A (CSA) found in the placental intervillous spaces ${ }^{9,10}$. The placental binding tropism is mediated by a single $P$. falciparum variant antigen, VAR2CSA, which is expressed at the surface of placental IEs ${ }^{11-14}$. VAR2CSA is an unusually strain-transcendent member of the highly polymorphic P. falciparum Erythrocyte Membrane Protein 1 (PfEMP1) family, present in one or more gene copies in every $P$. falciparum genotype ${ }^{15}$. VAR2CSA is a large multidomain protein consisting of six Duffy-binding like (DBL) domains (three DBLx, followed by three DBL $\varepsilon$ ). It also contains a $\mathrm{CIDR}_{\mathrm{PAM}}$ domain between the DBL2x and DBL3x domains, in a region that is also referred to as interdomain 2 (ID2). The core CSA-binding site in VAR2CSA has been mapped to the DBL2x domain and the flanking interdomain 1 (ID1) and ID2 regions ${ }^{16,17}$.

${ }^{1}$ Université de Paris, UMR_S1134, BIGR, INSERM, F-75015, Paris, France. ${ }^{2}$ Institut National de la Transfusion Sanguine, F-75015, Paris, France. 'Laboratory of excellence GR-Ex, F-75015, Paris, France. 'Seattle Children's Research Institute, Seattle, WA, 98109, USA. ${ }^{5}$ Department of Global Health, University of Washington, Seattle, WA, 98195, USA. *email: benoit.gamain@inserm.fr 
The N-terminal region of VAR2CSA stands today as a leading candidate for developing a placental malaria vaccine. Recombinant proteins based on the core CSA-binding site in VAR2CSA can elicit adhesion-blocking antibodies and pre-clinical assessments of the two most advanced VAR2CSA-based vaccine candidates PAMVAC and PRIMVAC (ClinicalTrials.gov identifiers NCT02647489 and NCT02658253, respectively) ${ }^{18}$ have paved the way for the clinical development of a vaccine that could protect women against $\mathrm{PM}^{19-21}$. However, it is unlikely that a single $P$. falciparum-derived VAR2CSA variant will be sufficient to induce a broad enough humoral response in humans to cross-react with the genetically diverse parasite populations present in endemic areas. Like other highly polymorphic vaccine targets, such as influenza and HIV, immuno-focusing vaccine strategies may be required to target antibodies on functionally conserved epitopes in VAR2CSA.

One of the closest $P$. falciparum relative is the chimpanzee parasite Plasmodium reichenowi ${ }^{22}$. Both parasite species belong to the Laverania sub-genus and share extensive gene organization and orthology, including the presence of var-like genes in $P$. reichenowi $i^{23,24}$. Although little is known about the encoded cytoadhesive properties of the var-like genes in most Laverania parasites, analysis of CIDR $\alpha 0$ and CIDR $\alpha 1$ recombinant domains from $P$. reichenowi has provided evidence that the CD36 and EPCR adhesion traits arose in an ancestral species ${ }^{24}$ prior to chimpanzee and human speciation. Moreover, genome sequencing of all known Laverania members revealed that var2csa is most likely a vestige of the ancestral gene family that has been maintained in $P$. falciparum and $P$. reichenowi ${ }^{25}$. Notably, the var $2 c s a$-like gene in the genome reference $P$. reichenowi CDC strain is annotated as a pseudogene and encodes a truncated protein (NTS-DBL1x-ID1-DBL2x-truncated ID2). In addition to its role in placental cytoadhesion, the $P$. falciparum var2csa gene has been proposed to be a central intermediate in var gene switching during antigenic variation ${ }^{26}$. The biological role(s) played by VAR2CSA in ape parasites and the potential clinical consequences for the natural host are still unknown. In order to investigate the evolutionary history of CSA-binding determinants, we performed a functional characterization of VAR2CSA from $P$. reichenowi (Pr-VAR2CSA) and also assessed the conservation of VAR2CSA antibody epitopes, which could have been conserved after evolutionary radiation of the Laverania sub-genus. We provide evidence that Pr-VAR2CSA binds to CSA with similar affinity and specificity as VAR2CSA from P. falciparum (Pf-VAR2CSA), and that it has the capability to elicit cross-inhibitory antibodies against different CSA-binding $P$. falciparum parasite lines.

\section{Results}

VAR2CSA from $P$. reichenowi binds to the placental receptor CSA with high specificity and affinity. The genome reference $P$. reichenowi CDC strain encodes a truncated VAR2CSA gene that has been annotated as a pseudogene. As compared to P. falciparum, the extracellular part of the protein is limited to the NTS-DBL1x-ID1-DBL2x-truncated ID2 (tID2) region (Fig. 1a). Since the CSA-binding region of $P$. falciparum VAR2CSA resides within the ID1-DBL2x-ID2a region of the protein ${ }^{27}$, we hypothesized that $P$. reichenowi VAR2CSA could also harbour functional determinants allowing interaction with glycosaminoglycans. In order to assess if $P$. reichenowi VAR2CSA presents a similar CSA-binding phenotype to that of VAR2CSA from $P$. falciparum, different Pr-VAR2CSA constructs were generated (Fig. 1a) and produced as soluble recombinant proteins in HEK cells (Fig. 1b). In addition to a recombinant protein comprising the whole extracellular part of Pr-VAR2CSA, three other protein constructs deprived of either the N-terminal sequence (Pr-DBL1x-ID2) or the tID2 region (Pr-NTS-DBL2x) or lacking both regions (Pr-DBL1x-2x) were designed. The full-length extracellular region of $P$. falciparum VAR2CSA (Pf-VAR2CSA) was also produced as well as three multi-domain constructs comprising the DBL1x-2x part of VAR2CSA and originating from different $P$. falciparum parasite strains (Pf-3D7-DBL1x-2x, Pf-FCR3-DBL1x-2x, Pf-7G8-DBL1x-2x) (Fig. 1a). The Pf-3D7-DBL1x-2x sequence boundaries (aa48-aa981) matches the exact same sequence delimitations of PRIMVAC (Pf-3D7-DBL1x-2x), a leading Pf-VAR2CSA-based PM vaccine currently evaluated in a phase Ia/Ib clinical trial ${ }^{19,21}$. A multiple sequence alignment performed with Pr-VAR2CSA and Pf-VAR2CSA (DBL1x-DBL2x) sequences from the 3D7, FCR3 and 7G8 strains revealed that the $P$. reichenowi protein presents $75.0 \%$ identity with Pf-3D7-VAR2CSA, 76.9\% with Pf-FCR3-VAR2CSA and 75.4\% with Pf-7G8-VAR (Supplementary Fig. S1).

Following a 2-step purification process comprising metallo-affinity and gel filtration chromatography, all recombinant proteins were successfully produced with a high degree of purity as reflected by sodium dodecyl sulphate-polyacrylamide gel electrophoresis (SDS-PAGE) (Fig. 1b). Even though protein integrity was excellent for most of the constructs, slight degradation bands were visible for Pr-DBL1x-tID2 following gel staining with Coomassie blue (Fig. 1b).

The glycosaminoglycan-binding properties of the different recombinant proteins derived from P. reichenowi VAR2CSA were then analysed by direct enzyme-linked immunosorbent assay (ELISA) in which chondroitin sulphate C (CSC), CSA and the CSA-bearing bovine glycoprotein decorin were immobilized on plastic. Bovine serum albumin (BSA) was also used as a non-glycosaminoglycan control protein. PrVAR2CSA, Pr-NTS-DBL2x and Pr-DBL1x-2x specifically bound to CSA and very poorly to CSC or BSA (Fig. 2). In contrast, Pr-DBL1x-tID2 displayed a non-negligible binding to CSC and BSA demonstrating a lower CSA-specificity, as compared to the other constructs. Recombinant protein binding to decorin was consistently higher than binding to purified CSA. Decorin was used in surface plasmon resonance (SPR) experiments to measure the association strength of the different constructs to CSA (Figs 3 and S2). For these experiments, BSA was coated on the reference channel $(\mathrm{Fc} 1)$ and decorin was immobilized on the analytic channel (Fc2). The specific binding response of the analytes towards decorin was regarded as the Fc2-Fc1 signal. The fitted kinetic data (Koff and Kon) derived from the sensorgrams (Supplementary Fig. S2) are displayed as a RaPID plot in Fig. 3. The affinity constant $\mathrm{K}_{\mathrm{D}}$ was regarded as Koff/Kon. Pr-VAR2CSA and Pr-DBL1x-2x demonstrated a nanomolar affinity to CSA $\left(\mathrm{K}_{\mathrm{D}}=70 \mathrm{nM}\right.$ and $82 \mathrm{nM}$, respectively), approaching the low nanomolar binding affinity of $P$. falciparum VAR2CSA for the sulphated sugar $\left(K_{\mathrm{D}}=16 \mathrm{nM}\right)$. In contrast, Pr-NTS-DBL2x had a much weaker interaction with CSA, as compared to the other construct $\left(\mathrm{K}_{\mathrm{D}}=247 \mathrm{nM}\right)$. Taken together, these results provide evidence that VAR2CSA from the chimpanzee malaria parasite $P$. reichenowi possesses functional determinants for specific CSA-binding. 
a

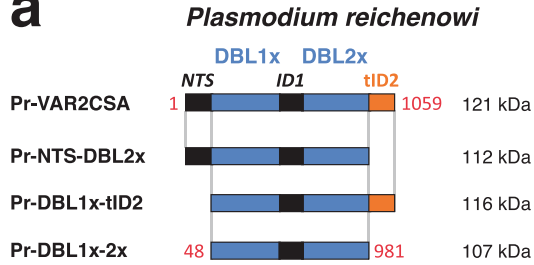

Plasmodium falciparum

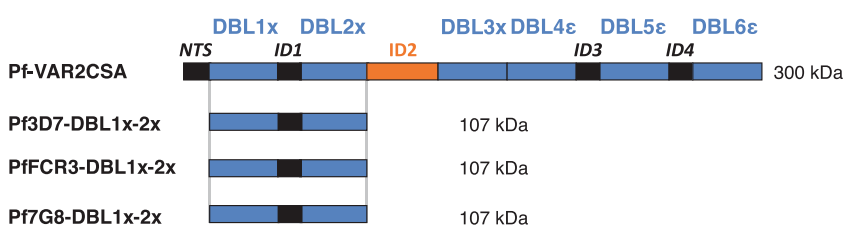

b

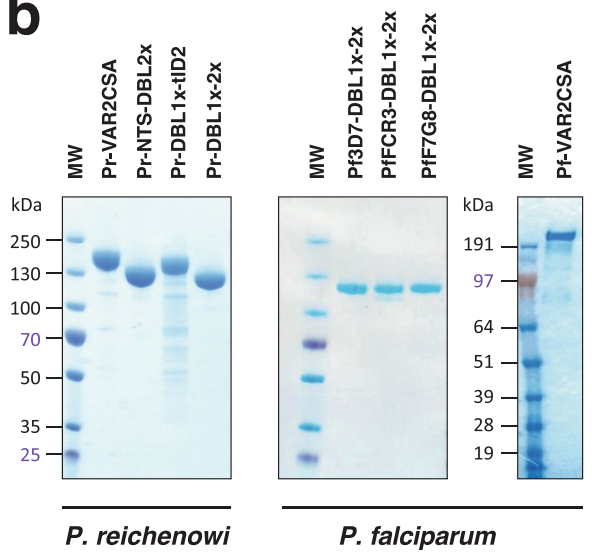

Figure 1. Recombinant proteins used in the study. (a) Schematic representation of the VAR2CSA protein organization and sequence boundaries of the multi-domain constructs used in this study. The extracellular part of VAR2CSA from P. reichenowi comprises a truncated gene encoding an NTS, two DBL domains (DBL1x to DBL2x) and a truncated ID2 (tID2). The first and last amino-acids of each construct are depicted as they appear within the exon 1 sequence of VAR2CSA from P. reichenowi. P. falciparum comprises an N-terminal sequence (NTS), six Duffy Binding-Like domains (DBL1x to DBL6ع) interspaced by four inter-domain regions (ID1 to ID4). The ID2 region includes the CIDR $_{\mathrm{PAM}}$ domain. (b) SDS-PAGE under reducing conditions followed by Coomassie blue staining of the VAR2CSA-derived purified proteins. His-tagged recombinant proteins were purified in a 2-phase process comprising metallo-affinity and gel filtration chromatography. MW: molecular weight. Three independent gels were run and are displayed.

Anti-Pr-VAR2CSA antibodies recognize VAR2CSA from different $P$. falciparum strains. The existence of CSA-binding determinants in $P$. reichenowi VAR2CSA raises the possibility that functionally conserved antigenic epitopes could exist between VAR2CSA from the chimpanzee parasite $P$. reichenowi and $P$. falciparum sequences. To investigate if cross-species epitopes are present, white rabbits and Wistar rats were immunized with recombinant Pr-VAR2CSA (NTS-DBL1x-ID1-DBL2x-tID2). IgG titrations performed 7 days after the last immunisation showed that Pr-VAR2CSA was more immunogenic in rats than in rabbits as reflected by higher anti-Pr-VAR2CSA IgG antibody titres in the rodent group (Table 1). Rat and rabbit anti-Pr-VAR2CSA antibodies cross-reacted on VAR2CSA-derived recombinant proteins (DBL1x-2x) originating from different $P$. falciparum sequences (3D7, FCR3 and 7G8 strains), thus implying the presence of cross-species epitopes in VAR2CSA. The levels of VAR2CSA-specific antibody obtained towards the $P$. falciparum DBL1x-2x constructs were much lower than Pr-VAR2CSA (10 to 100-fold) suggesting that cross-species epitopes are limited between the $P$. reichenowi and $P$. falciparum VAR2CSA sequences.

VAR2CSA immune recognition was then assessed in the context of the native VAR2CSA protein expressed at the surface of $P$. falciparum infected erythrocytes (IEs). Erythrocytes were infected with 3 different parasite lines selected for the CSA-binding phenotype (NF54-CSA, FCR3-CSA and 7G8-CSA) or for the CD36-binding phenotype (FCR3-CD36) as a negative control. The sequence of VAR2CSA from the NF54 strain is identical to the NF54-derived clone 3D7.

Flow cytometry analysis showed that IgGs present in rat plasma samples were able to recognize IEs expressing different VAR2CSA variants from P. falciparum parasites (Fig. 4a). The fold increase in fluorescence signal between immune and pre-immune sera was greater for NF54-CSA (mean fold increase: 11.4) than for FCR3-CSA (8.9) or 7G8-CSA (6.2). The capability of individual rat sera to recognize VAR2CSA varied between animals and 

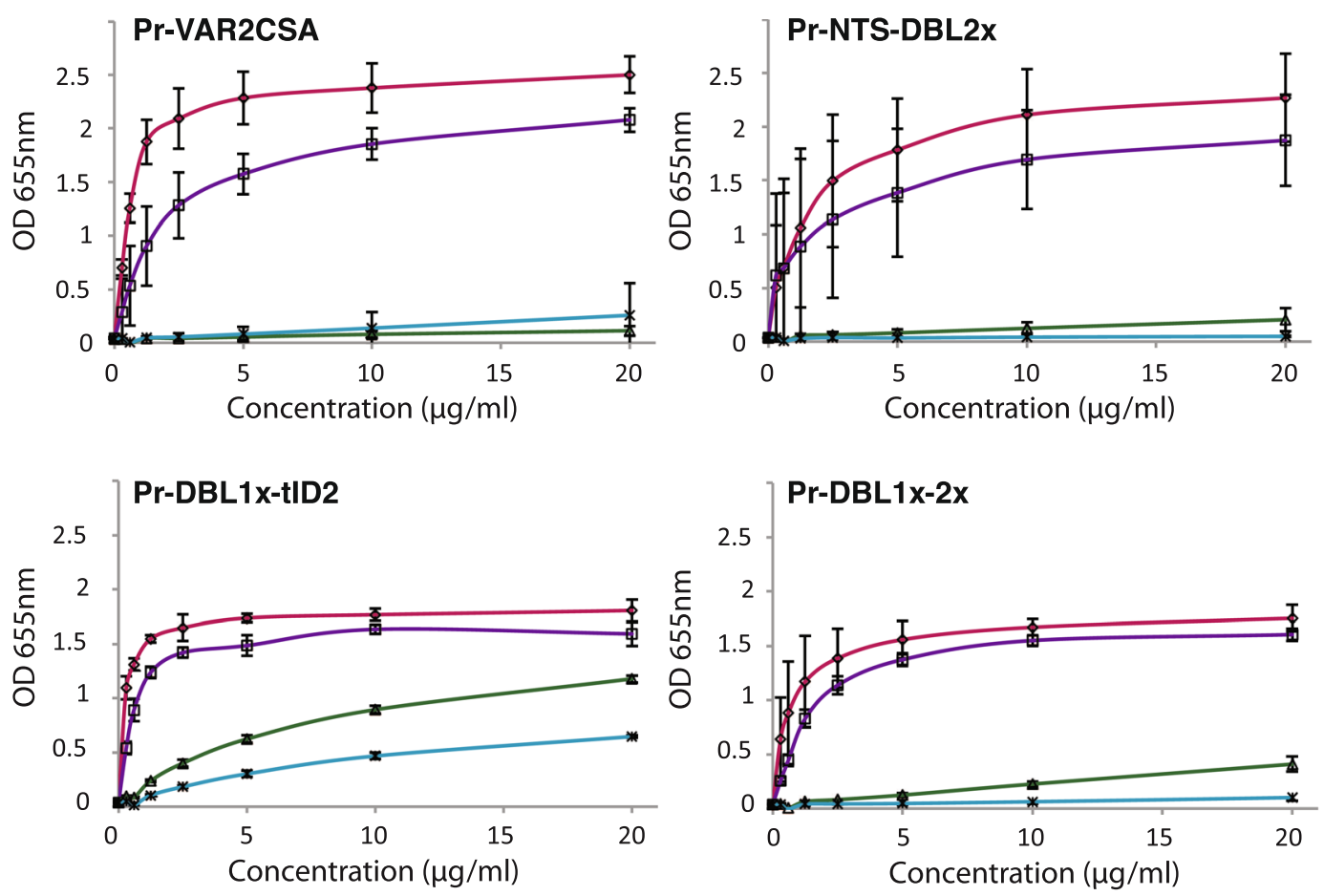

$\diamond$ Decorin $\rightarrow$ CSA $\triangle$ CSC $*$ BSA

Figure 2. VAR2CSA from P. reichenowi binds to the placental receptor CSA with high specificity. ELISA-based direct binding assay of the VAR2CSA-derived constructs to different sulphated glycosaminoglycans. Increasing concentrations of recombinant (a) Pr-VAR2CSA, (b) Pr-NTS-DBL2x, (c) Pr-DBL1x-tID2, (d) Pr-DBL1x-2x at serial dilutions of $0.31-20 \mu \mathrm{g} / \mathrm{mL}$ were added to wells previously coated with bovine serum albumin (BSA) or with different glycosaminoglycans; CSA-bearing bovine glycoprotein decorin, chondroitin sulphate A (CSA), chondroitin sulphate C (CSC). Error bars represent the standard deviation of 3 independent experiments.

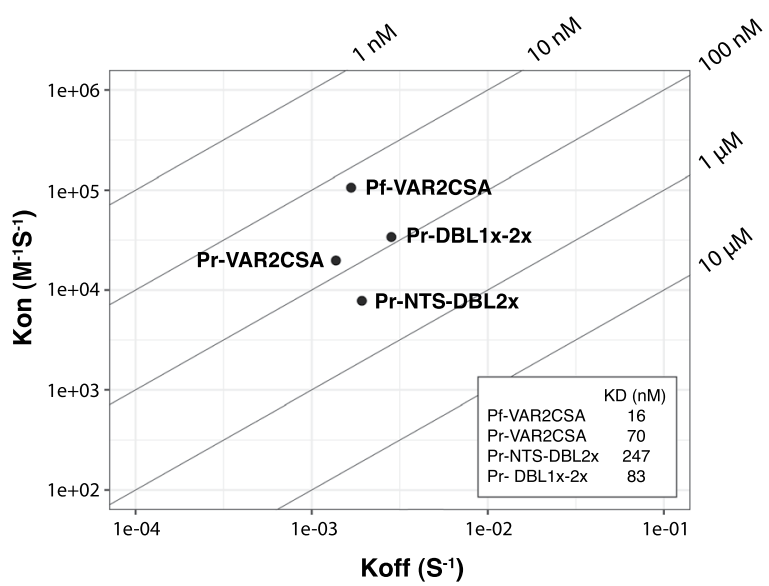

Figure 3. VAR2CSA from $P$. reichenowi binds to the placental receptor CSA with high affinity. RaPID plot of kinetics constants determined by surface plasmon resonance. Koff and Kon values for each analyte are indicated as dots in a 2-dimentional plot. Calculated $\mathrm{K}_{\mathrm{D}}$ values (Koff/Kon) are depicted in the inset table.

did not completely correlate with the antibody titres obtained using the matching DBL1x-2x recombinant proteins. The FCR3-CD36 IE population expressing a non-VAR2CSA PfEMP1 was not recognised by any of the rat samples (Fig. 4a). The rabbit anti-sera were also able to recognize native $P$. falciparum VAR2CSA variants and not the negative control FCR3-CD36 IEs, although at a lower level than the rat sera (two rabbits: mean fold increase NF54-CSA: 1.6; FCR3-CSA: 1.4; 7G8-CSA: 2.7) (Fig. 4b). These results demonstrate the presence of cross-species epitopes within VAR2CSA. 


\begin{tabular}{|l|l|l|l|l|}
\hline & Pr-VAR2CSA & $\begin{array}{l}\text { Pf3D7- } \\
\text { DBL1x-2x }\end{array}$ & $\begin{array}{l}\text { PfFCR3- } \\
\text { DBL1x-2x }\end{array}$ & $\begin{array}{l}\text { Pf7G8- } \\
\text { DBL1x-2x }\end{array}$ \\
\hline Rat 1 & 5297 & 323 & 298 & 515 \\
\hline Rat 2 & 43067 & 489 & 972 & 1925 \\
\hline Rat 3 & 3971 & 87 & 54 & 71 \\
\hline Mean (SD) & $17445(22199)$ & $300(202)$ & $441(475)$ & $837(968)$ \\
\hline Rabbit 1 & 1879 & 403 & 251 & 281 \\
\hline Rabbit 2 & 450 & 55 & 39 & 172 \\
\hline Mean (SD) & $1165(1010)$ & $229(246)$ & $145(150)$ & $227(77)$ \\
\hline
\end{tabular}

Table 1. IgG titers (1/) at Day 63.
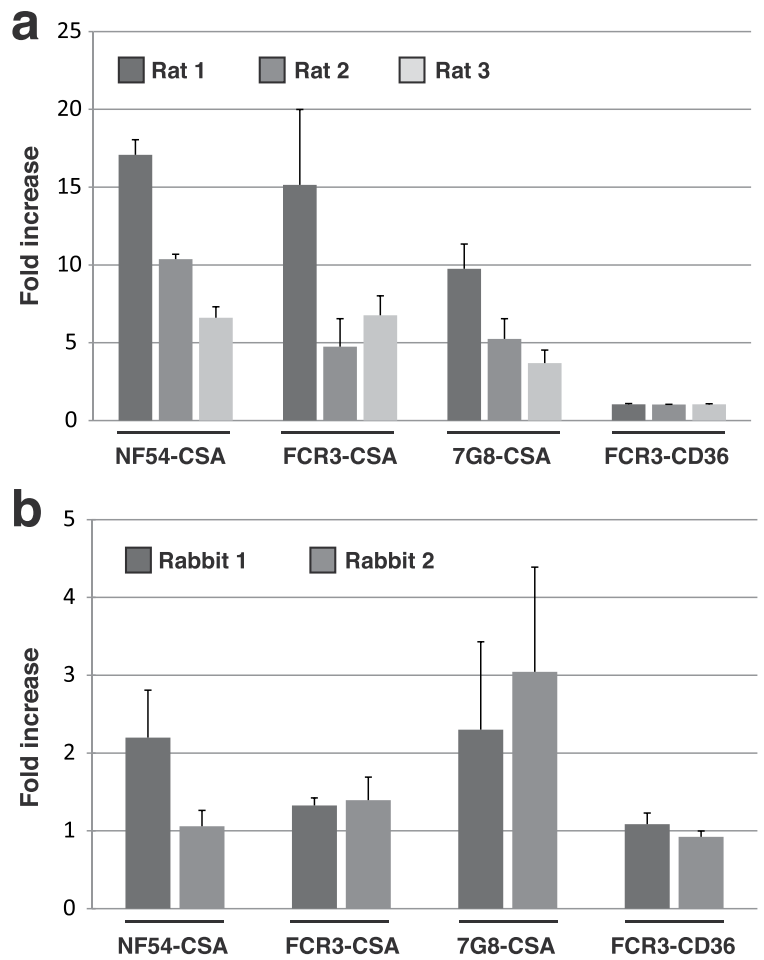

Figure 4. Anti-Pr-VAR2CSA antibodies recognize VAR2CSA from different P. falciparum strains. Immune recognition of erythrocytes infected by different $P$. falciparum parasite strains (NF54, FCR3, and 7G8) selected for different adhesive phenotypes (CSA and CD36) by immunisation-induced antibodies directed towards $P$. reichenowi VAR2CSA. Infected erythrocytes were incubated with rat sera (a) or rabbit sera (b) diluted 1:10. Erythrocyte-bound IgGs were detected using an anti-rat IgG or an anti-rabbit IgG specific antibody PEconjugated. Cells were then subjected to flow cytometry analysis. Results are expressed as the fold-increase in geometrical mean fluorescence intensity obtained with immune (Day 63) sera compared to pre-immune sera. Error bars represent the standard deviation of 3 independent experiments.

Anti-Pr-VAR2CSA antibodies inhibit the interaction between CSA and erythrocytes infected with different $P$. falciparum strains. The capacity of anti-Pr-VAR2CSA to block the interaction between CSA and P. falciparum-infected erythrocytes was assessed in CSA-binding inhibition assays (Fig. 5). The mean percentage of inhibition with rat anti-Pr-VAR2CSA sera was 37\% for NF54-CSA, 36\% for FCR3-CSA and 75\% for 7G8-CSA (Fig. 5a). The mean percentage of inhibition for the rabbit anti-sera was $40 \%$ for NF54-CSA, 15\% for FCR3-CSA and 38\% for 7G8-CSA (Fig. 5b). Taken together this demonstrates that Pr-VAR2CSA was capable of generating cross-species inhibitory antibodies.

\section{Discussion}

Cytoadhesion of $P$. falciparum infected erythrocytes to endothelial cells and the placenta is a major virulence determinant. The main cytoadhesion ligand in $P$. falciparum is the var gene family ${ }^{28}$. Increasing lines of evidence from genomic sequencing reveals that the var gene family arose in the Laveranian sub-genus that infects chimpanzees, gorillas, and humans. Within the Laveranian subgenus, clade A and clade B parasites have become specialized for different hosts ${ }^{23,25,29-31}$. While all Laverania species retain a two-exon var gene structure and are organized into subtelomeric or internal var genes, the adhesion domains have diversified 


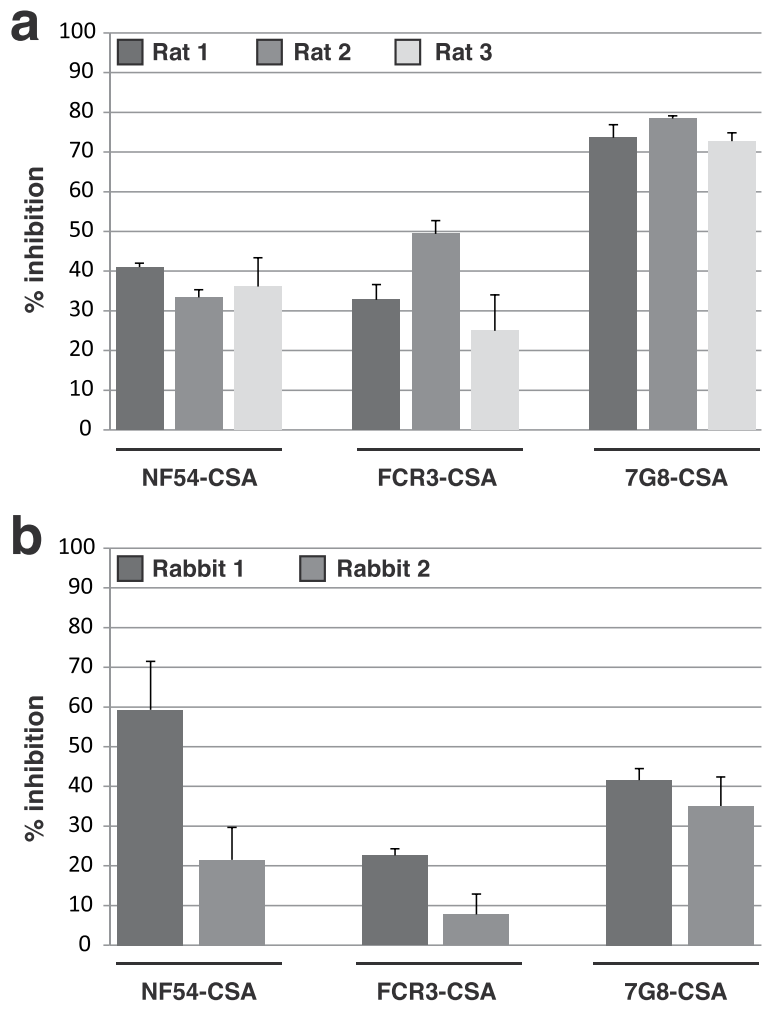

Figure 5. Anti-Pr-VAR2CSA antibodies inhibit the interaction between CSA and erythrocytes infected with different $P$. falciparum VAR2CSA strains. Inhibition of $P$. falciparum-infected erythrocytes binding to CSA by immunisation-induced antibodies directed towards Pr-VAR2CSA. Erythrocytes infected by different $P$. falciparum parasite strains (NF54, FCR3, and 7G8) selected for CSA adhesive phenotype were incubated with rat sera (a) or rabbit sera (b) diluted 1:10 and then plated on CSA-coated plates. CSA-binding inhibition was assessed by relative quantification of IEs remaining bound to the plate surface. Error bars represent the standard deviation of values obtained from three independent replicates.

between clade A and clade B parasites ${ }^{25}$. In particular, the var gene families of the human malaria parasite $P$. falciparum and the chimpanzee malaria parasite $P$. reichenowi highly resemble each other with similar DBL and CIDR sequence types, while different domain types are found in clade A Laverania parasites ${ }^{23,29-31}$. In contrast, var genes are completely absent in non-Laveranian plasmodia genomes. Taken together, genomic comparisons strongly suggest that the erythrocytic stage-related cytoadhesion traits originated as a unique adaptation in the Laverania sub-genus and then underwent adaptative radiation in different parasite-host combinations. Compared to $P$. falciparum, relatively little is known about the function and biological role of var genes in non-human primate hosts.

In P. falciparum, var genes have diverged to encode proteins that bind the endothelial receptors CD36 and $\mathrm{EPCR}^{8,32-34}$. Molecular analysis performed with recombinant CIDR domains from P. reichenowi revealed a similar interaction with the human receptors ${ }^{24}$, providing evidence that the CD36 and EPCR binding phenotypes evolved in an ancestral clade B parasite, and has been maintained as a key functional molecular interface in great apes and human hosts. Little is known about the evolutionary origins of other P. falciparum cytoadhesion traits.

Notably, nearly all Laverania species contain a var2csa-like gene ${ }^{25}$, suggesting an extremely ancient origin of this gene and it has been proposed to be a remnant of an ancient multigene family ${ }^{25}$. In $P$. falciparum, VAR2CSA is a placental adhesion ligand ${ }^{12}$ that is present in one or more gene copies in all parasite genotypes ${ }^{35,36}$, and the gene has also been proposed to function as a central intermediate when var genes switch during antigenic variation ${ }^{26}$. The core CSA binding region in P. falciparum VAR2CSA has been mapped to a region including both the DBLpam2 domain and the flanking ID1 and ID2/CIDRpam regions ${ }^{16,17}$. Although most Laverania parasites encode DBL and CIDR domains, the numbers of these domain types differ widely between Laverania species ${ }^{25}$. Consequently, the origins of the CSA-binding trait in the Laverania parasites and the function of var2csa-like gene in primate malaria parasites is unknown.

Here, we showed that $P$. reichenowi VAR2CSA recombinant proteins demonstrated a strong and specific interaction with CSA, comparable to the one of the P. falciparum orthologs. We observed similar binding results for different Pr-VAR2CSA constructs except for Pr-DBL1x-tID2 that lacked CSA-binding specificity and presented a weaker binding affinity for the sulphated sugar. However, this recombinant protein contained numerous truncated protein fragments, as compared to the other constructs. Since the Pr-DBL1x-2x recombinant protein presented a similar binding to the full-length Pr-VAR2CSA recombinant protein, it is unlikely that the sole absence of the NTS in Pr-DBL1x-tID2 is responsible for its reduced affinity and specificity. Instead, a structural misfolding due to inappropriate construct boundaries seems more probable to explain the altered Pr-DBL1x-tID2 function. 
Taken together, our results suggest that the VAR2CSA CSA-binding phenotype has an ancient origin and arose before Plasmodium falciparum crossed to humans.

An effective pregnancy malaria vaccine may need to elicit broadly cross-inhibitory antibodies against the VAR2CSA and CSA interaction. However, the extensive VAR2CSA sequence polymorphism poses problems, and there is still limited understanding of whether VAR2CSA contains conserved inhibitory epitopes or how to target them by vaccination. Previous work has shown that the core CSA-binding region in Pf-VAR2CSA (ID1-DBL2-ID2) can elicit adhesion-blocking epitopes, but the DBL2 domain contains several highly polymorphic loops that may contribute to immuno-evasion ${ }^{37}$. For other highly polymorphic vaccine targets, such as HIV and influenza, immuno-focusing vaccine strategies are being evaluated to induce broader neutralizing antibody responses. One strategy is cross-strain boosting with different allelic variants, which has also been evaluated for the CIDR domain in the PfEMP $1^{38,39}$. Here, we demonstrate that Pr-VAR2CSA immunogens are capable of eliciting cross-species inhibitory antibodies. While these epitopes did not dominate the antibody response, they may identify regions that have been functionally conserved between different VAR2CSA alleles. In the future, it will be interesting to characterize the cross-species inhibitory epitopes in Pr-VAR2CSA and to evaluate it in a cross-species boosting regimen to determine if this approach could enhance broadly inhibitory antibody responses.

\section{Methods}

VAR2CSA-derived proteins expression in HEK 293-F cells. The Pr-VAR2CSA pseudogene sequence encoding residues 1 to 1059 (PlasmoDB PRCDC_0011300) was synthetized with sequence optimization for human codon usage. All $\mathrm{N}$-glycosylation sites were mutated by substitution of the amino-acid asparagine with a glutamine. DNA sequences encoding for Pr-VAR2CSA, Pr-NTS-DBL2x (residues 1-981), Pr-DBL1x-tID2 (residues 48-1059) and Pr-DBL1X-2x (residues 48-981) were cloned into the expression plasmid pTT3. The Pf-VAR2CSA sequence was identical to the one previously reported ${ }^{40}$ and recombinant protein expression was carried out as previously described ${ }^{41}$. Briefly, FreeStyle 293-F cells (Invitrogen) were grown in Freestyle 293 serum free expression medium and transfected with the pTT3 plasmid containing the synthetic gene. Following a 72 hour-incubation at $37^{\circ} \mathrm{C}$ during which the recombinant protein was expressed and released in the medium, the cell culture was centrifuged and the supernatant was harvested for subsequent recombinant protein purification.

VAR2CSA-derived proteins expression in Escherichia coli SHuffle ${ }^{\circledR}$. DNA sequences encoding for the Pf-VAR2CSA multi-domains Pf-(3D7)-DBL1x-2x (residues 48-981; PlasmoDB PF3D7_1200600), Pf-(FCR3)-DBL1x-2x (residues 48-981; PlasmoDB PfIT_120006100), Pf-(7G8)-DBL1x-2x (residues 48-981; PlasmoDB Pf7G8_120005700) were amplified from genomic DNA of the P. falciparum strains 3D7, FCR3 (IT) and 7G8, respectively and cloned into pET-15b. Recombinant proteins were expressed in SHuffle ${ }^{\circledR}$ bacteria (New England Biolabs) for $20 \mathrm{~h}$ at $20^{\circ} \mathrm{C}$ following Isopropyl $\beta$-d-1-thiogalactopyranoside (IPTG) induction $(0.2 \mathrm{mM})$. All recombinant proteins carried a C-terminal His-tag and were purified in a 2-phase process comprising a metallo-affinity chromatography purification step performed on HisTrap ${ }^{\mathrm{TM}}$ columns (GE Healthcare) followed by a size exclusion chromatography step on a Superdex 200 10/300 GL column (GE Healthcare).

Enzyme-Linked Immunosorbent Assay (ELISA). ELISA were performed as previously described ${ }^{16}$. Briefly, microtiter plates (Immunolon $4 \mathrm{HBX} 3855$ ) were coated overnight at $4{ }^{\circ} \mathrm{C}$ with different sulfated glycosaminoglycans (GAG): $5 \mu \mathrm{g} / \mathrm{mL}$ for decorin (Sigma, D8428); $50 \mu \mathrm{g} / \mathrm{mL}$ for chondroitin sulfate A (CSA) (Sigma, C8529) and chondroitin sulfate C (CSC) (Sigma, 400670). After blocking, each recombinant protein (Pr-VAR2CSA, Pr-NTS-DBL2x, Pr-DBL1x-tID2 and Pr-DBL1x-2x) in serial dilutions of 0.31-20 $\mu \mathrm{g} / \mathrm{mL}$ was added into the wells and incubated for $1 \mathrm{~h}$ at $37^{\circ} \mathrm{C}$. His-tagged protein detection was performed using an anti-His HRP-conjugated antibody (Qiagen), diluted 1/2000. Absorbance was measured at $655 \mathrm{~nm}$ following addition of $\operatorname{TMB}\left(3,3^{\prime}, 5,5^{\prime}\right.$-tetramethylbenzidine) (Biorad).

Surface plasmon resonance. Interaction between the recombinant proteins and bovine decorin (Sigma, D8428) was studied by surface plasmon resonance (SPR) using a Biacore ${ }^{\circledR}$ X100 system (GE Healthcare). Bovine decorin was biotinylated using EZ-Link ${ }^{\mathrm{TM}}$ Sulfo-NHS-LC-Biotin kit (ThermoFischer Scientific) and immobilized on a SA sensor chip (GE Healthcare). The amount of immobilized decorin corresponded to 268 Response Units (RU). A separate flow channel on the same sensor chip, reserved for control runs, was prepared in the same way but with biotinylated BSA, immobilized at a level of $685 \mathrm{RU}$. Analytes were injected in dilution series at $25^{\circ} \mathrm{C}$ and at a flow rate of $20 \mu \mathrm{L} \cdot \mathrm{min}^{-1}$. Between each injection, surfaces were regenerated by 2 washes with $5 \mu \mathrm{L}$ of $2 \mathrm{M}$ $\mathrm{NaCl}$ followed by 1 wash with $1 \mathrm{M} \mathrm{NaCl} / 50 \mathrm{mM} \mathrm{NaOH}$. All curves were corrected for nonspecific binding by subtraction of control curves obtained from injection of the corresponding protein through the blank flow channel. Kinetic constants were determined following a 1:1 binding curve fitting.

Animal immunization. Small animal immunizations with the Pr-VAR2CSA recombinant protein were performed by Biotem (Grenoble, France) according to animal immunization guidelines. All animal immunization experiments were executed in strict accordance with good animal practices, following the EU animal welfare legislation and after approval of the Biotem (France) ethical committee. Every effort was made to minimize suffering.

Two white rabbits and three Wistar rats received each 4 injections of Pr-VAR2CSA (50 $\mu \mathrm{g}$ in PBS) in combination with Freund adjuvant following the immunization procedures described in Supplementary Fig. 3. Animals were bled 7 days after the last immunisation (day 63). Sera samples were then collected and stored at $-20^{\circ} \mathrm{C}$ until experimental analysis. 
IgG titers. IgG titers determination was performed as previously described ${ }^{21}$. Briefly, microtiter plates (Immunolon $4 \mathrm{HBX} 3855$ ) were coated overnight with $1 \mu \mathrm{g} / \mathrm{ml}$ antigen. After removal of blocking solution, rabbit or rat sera serial dilutions were added into the wells and incubated for $1 \mathrm{~h}$ at $37^{\circ} \mathrm{C}$. Anti-rabbit or anti-rat IgG (Fc-specific) HRP-conjugated antibody (Jackson Immunoresearch 111-036-046 or 112-036-071, respectively) at $1 / 2000$ were used for detection of bound-IgGs. Absorbance was measured at $655 \mathrm{~nm}$ following addition of TMB. After data plotting and 4-parameter logistic regression curve fitting, the plasma dilution corresponding to $50 \%$ of the maximal optical density (OD) value (sigmoid curve plateau) was regarded as the antigen specific antibody titer.

Parasite culture. Parasite culture was carried out as previously described ${ }^{21}$. Erythrocytes infected with NF54, FCR3 (IT4) and 7G8 were selected for the CSA-binding phenotype by multiple panning rounds on CSA (Sigma). These selected populations are referred to as NF54-CSA, FCR3-CSA and 7G8-CSA. Erythrocytes infected with FCR3 were also selected for the CD36-binding phenotype by multiple panning rounds on recombinant CD36 (R\&D systems) and are referred to as FCR3-CD36.

Flow cytometry. VarioMACS (Miltenyi Biotec) purified IEs at mid/late trophozoite stage were resuspended in PBS $0.2 \%$ BSA and counted. For each assay, $2.5 \times 10^{5}$ IEs were washed in PBS and incubated with the sera (previously depleted against normal erythrocytes) diluted $1 / 10$ in PBS $0.2 \%$ BSA for $1 \mathrm{~h}$ at $4{ }^{\circ} \mathrm{C}$. IEs were washed twice with PBS and resuspended in $100 \mu \mathrm{l}$ of PE conjugated goat anti-rabbit or goat anti-rat IgG diluted $1 / 100$ in PBS $0.2 \%$ BSA for $60 \mathrm{~min}$ at $4^{\circ} \mathrm{C}$. After washing twice in PBS $0.2 \% \mathrm{BSA}$, IEs were resuspended in paraformaldehyde $2 \%$ in PBS and kept at $4{ }^{\circ} \mathrm{C}$ overnight in darkness. Cells were then washed twice with PBS and analyzed by flow cytometry using a BD FACScanto II flow cytometer with the Flow Jo 10.0 software. Parasite nuclei were stained with Topro3 (1/4,000 dilution) (ThermoFischer Scientific). The results are expressed as the ratio between the geometric mean fluorescence intensities (MFI) of the immune sera and the respective non-immune sera.

CSA-binding inhibition assays. CSA-binding inhibition were performed as previously described ${ }^{21}$. Briefly, microtiter plates were coated overnight with CSA (Sigma) at $1 \mathrm{mg} / \mathrm{ml}$ or BSA (Roche) at $1 \%$. Purified infected erythrocytes (trophozoite stage) were pre-incubated with plasma samples diluted in RPMI $2 \%$ FBS $(1 / 10)$ for $1 \mathrm{~h}$ at $37^{\circ} \mathrm{C}$. Infected erythrocytes were then added into the pre-coated wells $\left(10^{6} \mathrm{cells} ; 100 \mu \mathrm{l} /\right.$ well $)$ and incubated for $1 \mathrm{~h}$ at $37^{\circ} \mathrm{C}$. Plates were then washed three times and infected erythrocytes remaining attached to the surface were lysed by addition of TMB. Absorbance was measured at $655 \mathrm{~nm}$. For rabbits, results were expressed as \% of inhibition of the post-immunization samples compared to the respective pre-immunization samples. Due to limited amounts of pre-immune samples, rat results were expressed as \% of inhibition of the post-immunization samples compared to medium alone.

\section{Data availability}

The authors confirm that the data supporting the findings of this study are available within the article and its Supplementary Material.

Received: 3 July 2019; Accepted: 24 October 2019;

Published online: 18 November 2019

\section{References}

1. Walter, P. R., Garin, Y. \& Blot, P. Placental pathologic changes in malaria. A histologic and ultrastructural study. Am J Pathol 109, 330-342 (1982).

2. Galbraith, R. M., Faulk, W. P., Galbraith, G. M., Holbrook, T. W. \& Bray, R. S. The human materno-foetal relationship in malaria: I. Identification of pigment and parasites in the placenta. Trans R Soc Trop Med Hyg 74, 52-60 (1980).

3. Galbraith, R. M. et al. The human materno-foetal relationship in malaria. II. Histological, ultrastructural and immunopathological studies of the placenta. Trans R Soc Trop Med Hyg 74, 61-72 (1980).

4. Brabin, B. J. et al. The sick placenta-the role of malaria. Placenta 25, 359-378, https://doi.org/10.1016/j.placenta.2003.10.019 (2004).

5. Fried, M., Nosten, F., Brockman, A., Brabin, B. J. \& Duffy, P. E. Maternal antibodies block malaria. Nature 395, 851-852, https://doi. org/10.1038/27570 (1998).

6. Barnwell, J. W. et al. A human 88-kD membrane glycoprotein (CD36) functions in vitro as a receptor for a cytoadherence ligand on Plasmodium falciparum-infected erythrocytes. J Clin Invest 84, 765-772, https://doi.org/10.1172/JCI114234 (1989).

7. Berendt, A. R., Simmons, D. L., Tansey, J., Newbold, C. I. \& Marsh, K. Intercellular adhesion molecule-1 is an endothelial cell adhesion receptor for Plasmodium falciparum. Nature 341, 57-59, https://doi.org/10.1038/341057a0 (1989).

8. Turner, L. et al. Severe malaria is associated with parasite binding to endothelial protein C receptor. Nature 498, 502-505, https:// doi.org/10.1038/nature12216(2013).

9. Fried, M. \& Duffy, P. E. Maternal malaria and parasite adhesion. J Mol Med (Berl) 76, 162-171 (1998).

10. Rogerson, S. J. \& Beeson, J. G. The placenta in malaria: mechanisms of infection, disease and foetal morbidity. Ann Trop Med Parasitol 93(Suppl 1), S35-42 (1999).

11. Salanti, A. et al. Selective upregulation of a single distinctly structured var gene in chondroitin sulphate A-adhering Plasmodium falciparum involved in pregnancy-associated malaria. Mol Microbiol 49, 179-191 (2003).

12. Salanti, A. et al. Evidence for the involvement of VAR2CSA in pregnancy-associated malaria. J Exp Med 200, 1197-1203, https://doi. org/10.1084/jem.20041579 (2004).

13. Viebig, N. K. et al. A single member of the Plasmodium falciparum var multigene family determines cytoadhesion to the placental receptor chondroitin sulphate A. EMBO Rep 6, 775-781, https://doi.org/10.1038/sj.embor.7400466 (2005).

14. Viebig, N. K. et al. Disruption of var2csa gene impairs placental malaria associated adhesion phenotype. PLoS One 2, e910, https:// doi.org/10.1371/journal.pone.0000910 (2007).

15. Sander, A. F. et al. Positive selection of Plasmodium falciparum parasites with multiple var2csa-type PfEMP1 genes during the course of infection in pregnant women. J Infect Dis 203, 1679-1685, https://doi.org/10.1093/infdis/jir168 (2011).

16. Srivastava, A. et al. Var2CSA minimal CSA binding region is located within the N-terminal region. PLoS One 6, e20270, https://doi. org/10.1371/journal.pone.0020270 (2011). 
17. Dahlback, M. et al. The chondroitin sulfate A-binding site of the VAR2CSA protein involves multiple N-terminal domains. J Biol Chem 286, 15908-15917, https://doi.org/10.1074/jbc.M110.191510 (2011).

18. Chene, A. et al. Clinical development of placental malaria vaccines and immunoassays harmonization: a workshop report. Malar J 15, 476, https://doi.org/10.1186/s12936-016-1527-8 (2016).

19. Chene, A. et al. Down-selection of the VAR2CSA DBL1-2 expressed in E. coli as a lead antigen for placental malaria vaccine development. NPJ Vaccines 3, 28, https://doi.org/10.1038/s41541-018-0064-6 (2018).

20. Nielsen, M. A. et al. The Influence of Sub-Unit Composition and Expression System on the Functional Antibody Response in the Development of a VAR2CSA Based Plasmodium falciparum Placental Malaria Vaccine. PLoS One 10, e0135406, https://doi. org/10.1371/journal.pone.0135406 (2015).

21. Chene, A. et al. Preclinical immunogenicity and safety of the cGMP-grade placental malaria vaccine PRIMVAC. EBioMedicine. https://doi.org/10.1016/j.ebiom.2019.03.010 (2019).

22. Liu, W. et al. Origin of the human malaria parasite Plasmodium falciparum in gorillas. Nature 467, 420-425, https://doi.org/10.1038/ nature09442 (2010).

23. Larremore, D. B. et al. Ape parasite origins of human malaria virulence genes. Nat Commun 6, 8368, https://doi.org/10.1038/ ncomms9368 (2015).

24. Brazier, A. J., Avril, M., Bernabeu, M., Benjamin, M. \& Smith, J. D. Pathogenicity Determinants of the Human Malaria Parasite Plasmodium falciparum Have Ancient Origins. $m$ Sphere 2, https://doi.org/10.1128/mSphere.00348-16 (2017).

25. Otto, T. D. et al. Genomes of all known members of a Plasmodium subgenus reveal paths to virulent human malaria. Nat Microbiol 3, 687-697, https://doi.org/10.1038/s41564-018-0162-2 (2018).

26. Ukaegbu, U. E. et al. A Unique Virulence Gene Occupies a Principal Position in Immune Evasion by the Malaria Parasite Plasmodium falciparum. PLoS Genet 11, e1005234, https://doi.org/10.1371/journal.pgen.1005234 (2015).

27. Clausen, T. M. et al. Structural and functional insight into how the Plasmodium falciparum VAR2CSA protein mediates binding to chondroitin sulfate A in placental malaria. J Biol Chem 287, 23332-23345, https://doi.org/10.1074/jbc.M112.348839 (2012).

28. Miller, L. H., Baruch, D. I., Marsh, K. \& Doumbo, O. K. The pathogenic basis of malaria. Nature 415, 673-679, https://doi. org/10.1038/415673a (2002).

29. Otto, T. D. et al. Genome sequencing of chimpanzee malaria parasites reveals possible pathways of adaptation to human hosts. Nat Commun 5, 4754, https://doi.org/10.1038/ncomms5754 (2014).

30. Sundararaman, S. A. et al. Genomes of cryptic chimpanzee Plasmodium species reveal key evolutionary events leading to human malaria. Nat Commun 7, 11078, https://doi.org/10.1038/ncomms11078 (2016).

31. Zilversmit, M. M. et al. Hypervariable antigen genes in malaria have ancient roots. BMC Evol Biol 13, 110, https://doi. org/10.1186/1471-2148-13-110 (2013).

32. Lau, C. K. et al. Structural conservation despite huge sequence diversity allows EPCR binding by the PfEMP1 family implicated in severe childhood malaria. Cell Host Microbe 17, 118-129, https://doi.org/10.1016/j.chom.2014.11.007 (2015).

33. Robinson, B. A., Welch, T. L. \& Smith, J. D. Widespread functional specialization of Plasmodium falciparum erythrocyte membrane protein 1 family members to bind CD36 analysed across a parasite genome. Mol Microbiol 47, 1265-1278 (2003).

34. Avril, M., Brazier, A. J., Melcher, M., Sampath, S. \& Smith, J. D. DC8 and DC13 var genes associated with severe malaria bind avidly to diverse endothelial cells. PLoS Pathog 9, e1003430, https://doi.org/10.1371/journal.ppat.1003430 (2013).

35. Trimnell, A. R. et al. Global genetic diversity and evolution of var genes associated with placental and severe childhood malaria. Mol Biochem Parasitol 148, 169-180, https://doi.org/10.1016/j.molbiopara.2006.03.012 (2006).

36. Sander, A. F. et al. Multiple var2csa-type PfEMP1 genes located at different chromosomal loci occur in many Plasmodium falciparum isolates. PLoS One 4, e6667, https://doi.org/10.1371/journal.pone.0006667 (2009).

37. Bockhorst, J. et al. Structural polymorphism and diversifying selection on the pregnancy malaria vaccine candidate VAR2CSA. Mol Biochem Parasitol 155, 103-112, https://doi.org/10.1016/j.molbiopara.2007.06.007 (2007).

38. Gratepanche, S. et al. Induction of crossreactive antibodies against the Plasmodium falciparum variant protein. Proc Natl Acad Sci USA 100, 13007-13012, https://doi.org/10.1073/pnas.2235588100 (2003).

39. Baruch, D. I., Gamain, B. \& Miller, L. H. DNA immunization with the cysteine-rich interdomain region 1 of the Plasmodium falciparum variant antigen elicits limited cross-reactive antibody responses. Infect Immun 71, 4536-4543, https://doi.org/10.1128/ iai.71.8.4536-4543.2003 (2003).

40. Srivastava, A. et al. Full-length extracellular region of the var2CSA variant of PfEMP1 is required for specific, high-affinity binding to CSA. Proc Natl Acad Sci USA 107, 4884-4889, https://doi.org/10.1073/pnas.1000951107 (2010).

41. Srivastava, A., Durocher, Y. \& Gamain, B. Expressing full-length functional PfEMP1 proteins in the HEK293 expression system. Methods Mol Biol 923, 307-319, https://doi.org/10.1007/978-1-62703-026-7_22 (2013).

\section{Acknowledgements}

This work is supported by the French National Research Agency (ANR-16-CE11-0014-01), grants from Laboratory of Excellence GR-Ex, reference ANR-11-LABX-0051. The labex GR-Ex is funded by the program "Investissements d'avenir" of the French National Research Agency, reference ANR-11-IDEX-0005-02.

\section{Author contributions}

S.G., A.C., A.S. and B.G. conceived and designed the experiments. S.G., A.C., S.D. and A.S. performed the experiments. S.G., A.C. and B.G. analysed the data. M.A. and J.D.S. contributed to DNA sequence analysis and provided reagents/materials. S.G., A.C., J.D.S. and B.G. wrote the manuscript. All authors have read and approved the final version of the manuscript.

\section{Competing interests}

The authors declare no competing interests.

\section{Additional information}

Supplementary information is available for this paper at https://doi.org/10.1038/s41598-019-53334-8.

Correspondence and requests for materials should be addressed to B.G.

Reprints and permissions information is available at www.nature.com/reprints.

Publisher's note Springer Nature remains neutral with regard to jurisdictional claims in published maps and institutional affiliations. 
(c) (i) Open Access This article is licensed under a Creative Commons Attribution 4.0 International License, which permits use, sharing, adaptation, distribution and reproduction in any medium or format, as long as you give appropriate credit to the original author(s) and the source, provide a link to the Creative Commons license, and indicate if changes were made. The images or other third party material in this article are included in the article's Creative Commons license, unless indicated otherwise in a credit line to the material. If material is not included in the article's Creative Commons license and your intended use is not permitted by statutory regulation or exceeds the permitted use, you will need to obtain permission directly from the copyright holder. To view a copy of this license, visit http://creativecommons.org/licenses/by/4.0/.

(C) The Author(s) 2019 